Check for updates

Cite this: RSC Adv., 2020, 10, 31316

DOI: $10.1039 /$ dOra90087g

rsc.li/rsc-advances

\section{Correction: Toward realistic computer modeling of paraffin-based composite materials: critical assessment of atomic-scale models of paraffins}

\author{
Igor V. Volgin, (D) Artyom D. Glova, (D) Victor M. Nazarychev, (D) Sergey V. Larin, (iD \\ Sergey V. Lyulin (iD) and Andrey A. Gurtovenko (D)*
}

Correction for 'Toward realistic computer modeling of paraffin-based composite materials: critical assessment of atomic-scale models of paraffins' by Artyom D. Glova et al., RSC Adv., 2019, 9, 3883438847, DOI: 10.1039/C9RA07325F.

The authors regret that the 1-4 Lennard-Jones interactions were not accounted for in the molecular dynamics simulations of $n$-eicosane samples with the use of the united-atom GROMOS force field, as required by the original parametrization of this force field. ${ }^{1}$ This led to the abnormal behavior of the corresponding systems, namely: the $n$-eicosane samples did not crystallize within the temperature range of 200-450 K. After the 1-4 interactions were turned on, the GROMOS force field allowed us to observe the crystallization of $n$-eicosane with the transition temperature of $270 \pm 1 \mathrm{~K}$, see Fig. 1.

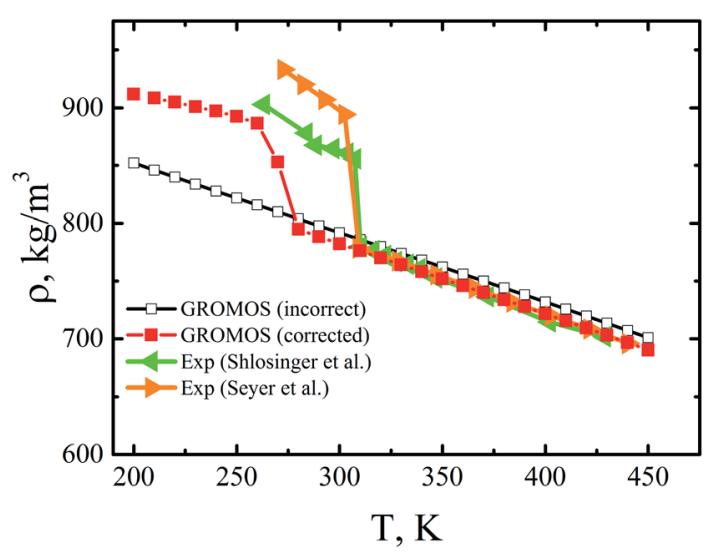

Fig. 1 Mass density of $n$-eicosane samples as a function of temperature for the GROMOS force field, cf. Fig. 2(b) of the original publication. The open black squares show the original incorrect data, and the closed red squares correspond to the corrected results. For the sake of comparison, the experimental data ${ }^{2,3}$ is also presented.

Most structural and dynamic characteristics also changed after the 1-4 interactions were properly accounted for. Below we present the updated versions of Tables 1 and 2 of the original publication.

Overall, the GROMOS force field does not show abnormal behavior for the $n$-eicosane sample anymore and performs rather similarly to other united-atom force fields such as e.g. PYS. The main conclusions of the original publication remain unchanged since the use of all-atom general-purpose force fields was shown to provide a more realistic description for $n$-eicosane as compared to their united-atom counterparts. 
Table 1 The crystallization temperature $\left(T_{c}\right)$, the coefficient of volumetric thermal expansion (CTE) measured in the temperature interval $400-$ $450 \mathrm{~K}$, the radius of gyration $\left(R_{\mathrm{g}}\right)$, and the end-to-end distance $H$ of $n$-eicosane chains for different force fields. The corrected data for the GROMOS force field is highlighted in bold

\begin{tabular}{lllllll}
\hline Force field & $T_{\mathrm{c}}, \mathrm{K}$ & $\mathrm{CTE}, 10^{-4} \mathrm{~K}^{-1}$ & $R_{\mathrm{g}}, \mathrm{nm}(T=250 \mathrm{~K})$ & $R_{\mathrm{g}}, \mathrm{nm}(T=450 \mathrm{~K})$ & $H, \mathrm{~nm}(T=250 \mathrm{~K})$ & $H, \mathrm{~nm}(T=450 \mathrm{~K})$ \\
\hline GAFF & $330 \pm 1$ & $17.4 \pm 0.1$ & $0.75 \pm 0.01$ & $0.60 \pm 0.01$ & $2.43 \pm 0.01$ & $1.70 \pm 0.01$ \\
GAFF2 & $365 \pm 2$ & $14.3 \pm 0.1$ & $0.75 \pm 0.01$ & $0.62 \pm 0.01$ & $2.43 \pm 0.01$ & $1.82 \pm 0.01$ \\
OPLS-AA & $365 \pm 1$ & $14.7 \pm 0.1$ & $0.74 \pm 0.01$ & $0.62 \pm 0.01$ & $2.42 \pm 0.01$ & $1.80 \pm 0.01$ \\
L-OPLS-AA & $265 \pm 3$ & $12.3 \pm 0.1$ & $0.72 \pm 0.01$ & $0.57 \pm 0.01$ & $2.29 \pm 0.01$ & $1.63 \pm 0.01$ \\
CHARMM36 & $320 \pm 1$ & $12.4 \pm 0.1$ & $0.74 \pm 0.01$ & $0.59 \pm 0.01$ & $2.39 \pm 0.01$ & $1.69 \pm 0.01$ \\
GROMOS & $\mathbf{2 7 0} \pm \mathbf{1}$ & $\mathbf{9 . 1} \pm \mathbf{0 . 1}$ & $\mathbf{0 . 7 2} \pm \mathbf{0 . 0 1}$ & $\mathbf{0 . 5 8} \pm \mathbf{0 . 0 1}$ & $\mathbf{2 . 3 4} \pm \mathbf{0 . 0 1}$ & $\mathbf{1 . 6 6} \pm \mathbf{0 . 0 1}$ \\
NERD & $270 \pm 1$ & $11.6 \pm 0.1$ & $0.74 \pm 0.01$ & $0.58 \pm 0.01$ & $2.41 \pm 0.01$ & $1.64 \pm 0.01$ \\
OPLS-UA & $310 \pm 1$ & $7.6 \pm 0.1$ & $0.73 \pm 0.01$ & $0.57 \pm 0.01$ & $2.38 \pm 0.01$ & $1.63 \pm 0.01$ \\
PYS & $270 \pm 10$ & $9.0 \pm 0.1$ & $0.72 \pm 0.01$ & $0.55 \pm 0.01$ & $2.34 \pm 0.01$ & $1.57 \pm 0.01$ \\
TraPPE & $280 \pm 1$ & $9.6 \pm 0.1$ & $0.74 \pm 0.01$ & $0.58 \pm 0.01$ & $2.41 \pm 0.01$ & 2.43 (ref. 5) \\
Experiment & 310 (ref. 4) & $8.8-8.9$ (ref. 2 and 3) & - & - & & -
\end{tabular}

Table 2 The shear viscosity, the diffusion coefficient and the mass density for $n$-eicosane samples in the liquid state simulated with different force fields at $T=450 \mathrm{~K}$. The corrected data for the GROMOS force field is highlighted in bold

\begin{tabular}{llll}
\hline Force field & $\eta, \mathrm{mPa} \mathrm{s}$ & $D, 10^{-5} \mathrm{~cm}^{2} \mathrm{~s}^{-1}$ & $\rho, \mathrm{kg} \mathrm{m} \mathrm{m}^{-3}$ \\
\hline GAFF & $0.42 \pm 0.04$ & $2.9 \pm 0.2$ & $592.3 \pm 0.1$ \\
GAFF2 & $0.59 \pm 0.01$ & $2.1 \pm 0.1$ & $633.5 \pm 0.1$ \\
OPLS-AA & $0.83 \pm 0.07$ & $1.6 \pm 0.1$ & $668.2 \pm 0.1$ \\
L-OPLS-AA & $0.64 \pm 0.08$ & $2.2 \pm 0.2$ & $656.4 \pm 0.1$ \\
CHARMM36 & $0.60 \pm 0.04$ & $2.1 \pm 0.2$ & $658.1 \pm 0.1$ \\
GROMOS & $\mathbf{0 . 5 2} \pm \mathbf{0 . 0 1}$ & $2.7 \pm \mathbf{0 . 1}$ & $\mathbf{6 9 0 . 3} \pm \mathbf{0 . 1}$ \\
NERD & $0.32 \pm 0.01$ & $3.6 \pm 0.1$ & $661.0 \pm 0.1$ \\
OPLS-UA & $0.79 \pm 0.02$ & $1.8 \pm 0.1$ & $753.1 \pm 0.1$ \\
PYS & $0.53 \pm 0.01$ & $2.6 \pm 0.1$ & $698.8 \pm 0.1$ \\
TraPPE & $0.45 \pm 0.04$ & $2.9 \pm 0.1$ & $693.7 \pm 0.1$ \\
Experiment & $0.594(T=453 \mathrm{~K})^{6}$ & $2.2(T=443 \mathrm{~K})^{7}$ & $696.0(T=440 \mathrm{~K})^{3}$
\end{tabular}

The Royal Society of Chemistry apologises for these errors and any consequent inconvenience to authors and readers.

\section{References}

1 L. D. Schuler, X. Daura and W. F. van Gunsteren, J. Comput. Chem., 2001, 22, 1205-1218.

2 A. P. Shlosinger and E. W. Bentilla, Research and development study on thermal control by use offusible materials, NASA Technical Report, NASA-CR-67695, 1965.

3 W. F. Seyer, R. F. Patterson and J. L. Keays, J. Am. Chem. Soc., 1944, 66, 179-182.

4 A. Sharma, V. V. Tyagi, C. R. Chen and D. Buddhi, Renewable Sustainable Energy Rev., 2009, 13, 318-345.

5 M. Rubinstein and R. H. Colby, Polymer physics, Oxford University Press, Oxford, 2003.

6 P. H. Gross and H. K. Zimmerman, Rheol. Acta, 1964, 3, 290-294.

7 E. von Meerwall, S. Beckman, J. Jang and W. L. Mattice, J. Chem. Phys., 1998, 108, 4299-4304. 